\title{
Curcumin Attenuates Chronic Unpredictable Mild Stress-Induced Depressive-Like Behaviors via Restoring Changes in Oxidative Stress and the Activation of Nrf2 Signaling Pathway in Rats
}

\author{
Dehua Liao, ${ }^{1}$ Chuanfeng Lv, ${ }^{2}$ Lizhi Cao, ${ }^{1}$ Dunwu Yao, ${ }^{1}$ Yi Wu, ${ }^{1}$ Minghui Long, ${ }^{1}$ Ni Liu $\mathbb{D}^{1},{ }^{1}$ \\ and Pei Jiang $\mathbb{( D}^{2}$ \\ ${ }^{1}$ Department of Pharmacy, Hunan Cancer Hospital, Changsha, 410013 Hunan, China \\ ${ }^{2}$ Institute of Clinical Pharmacy \& Pharmacology, Jining First People's Hospital, Jining, 272000 Shandong, China \\ Correspondence should be addressed to Ni Liu; liuni@hnca.org.cn and Pei Jiang; jiangpeicsu@sina.com
}

Received 14 April 2020; Revised 21 August 2020; Accepted 27 August 2020; Published 18 September 2020

Academic Editor: Francisco Jaime B. Mendonça Junior

Copyright (c) 2020 Dehua Liao et al. This is an open access article distributed under the Creative Commons Attribution License, which permits unrestricted use, distribution, and reproduction in any medium, provided the original work is properly cited.

\begin{abstract}
Accumulating evidence has demonstrated that oxidative stress is associated with depression. Our present study aimed at investigating the antidepressant effect and the possible mechanisms of curcumin (CUR) in chronic unpredictable mild stress(CUMS-) induced depression model in rats. After exposure to CUMS for four weeks, the rats showed depressive-like behavior, and the depressive-like behaviors in CUMS-treated rats were successfully corrected after administration of CUR. In addition, CUR could effectively decrease protein expression of oxidative stress markers (Nox2, 4-HNE, and MDA) and increase the activity of CAT. CUR treatment also reversed CUMS-induced inhibition of Nrf2-ARE signaling pathway, along with increasing the mRNA expression of NQO-1 and HO-1. Furthermore, the supplementation of CUR also increased the ratio of pCREB/CREB and synaptic-related protein (BDNF, PSD-95, and synaptophysin). In addition, CUR could effectively reverse CUMS-induced reduction of spine density and total dendritic length. In conclusion, the study revealed that CUR relieves depressive-like state through the mitigation of oxidative stress and the activation of Nrf2-ARE signaling pathway.
\end{abstract}

\section{Introduction}

As one of the most common neuropsychiatric illness, depression has affected 300 million people of all ages in the modern world [1]. According to the WHO's prediction, depression is expected to become the world's second leading cause of disability by 2020 [2], leading to a huge social and economic burden on the modern society [3]. In currently clinical practice, many chemical treatments are used for depression, such as tricyclic antidepressants, monoamine oxidase inhibitors, and selective serotonin reuptake inhibitors $[4,5]$. However, the existing treatments were not effective to all patients [6] and also accompanied with unwanted side effects $[7,8]$. Thus, it is necessary to develop a more effective and safer pharmacological intervention.

Increasing evidence suggested that oxidative stress is responsible for the development of depression [9]. Oxidative stress mainly focused on brain which has a limited amount of antioxidant capacity [10]. It was reported that antidepressants could effectively reduced oxidative damage in depressed patients [11, 12]. The antioxidant subjects like polyphenolic compounds exhibit antidepressant activity in experimentally induced depression models by modulating the brain oxidative stress status [13].

Oxidative stress is an etiologic factor in depressive/ neurodegenerative disorders that it is often accompanied by deregulation of nuclear factor erythroid-2-related factor 2 (Nrf2) pathway, a key antioxidant mechanism indicated as a promising target for treatment of depression [14]. As a pivotal transcription factor, Nrf2 was involved in the regulation of the antioxidant response in the brain. Under oxidative stress circumstances, Nrf2 isolate from Kelch-like ECHassociated protein 1 (Keap1) and translocate from cytoplasm into the nucleus $[15,16]$. Furthermore, antioxidant response element (ARE) could be upregulated after the activation of the Nrf2 and finally regulates the expressions of a variety 
of antioxidant enzymes like heme oxygenase-1 (HO-1) and NADPH: quinine oxidoreductase-1 (NQO-1) [17, 18]. Buendia et al. [19] has reported that Nrf2-ARE pathway is proved to reduce oxidative stress and neuroinflammation and play a protective role in neurodegenerative diseases.

Numerous evidence has indicated that depression was associated with a range of changes in synaptic form [20, 21]. Cyclic AMP response element-binding protein (CREB) was a major transcription factor involved in the regulation of genes associated with synaptic and neural plasticity. As an important neurotrophic factor, brain-derived neurotrophic factor (BDNF) supports growth and survival of neurons. Recent study has showed that CREB-BDNF signaling pathway in hippocampus was closely related to depression and the pathogenesis of cognitive function impairments [22]. PSD-95 and synaptophysin were postsynaptic marker and presynaptic marker, respectively, which play an important role in the maintenance of synaptic plasticity.

Curcumin (CUR) is the major active component extracted from Curcuma longa, which exhibited anti-inflammatory, antioxidant, immunomodulatory, and neuroprotective activities $[23,24]$. It has been increasingly recognized that CUR has the potential to cross blood brain barrier and exert antidepressant-like action. Xu et al. have reported that chronic administration of CUR produced a significant antidepressant property in the treatment of depression in mice model [25]. Notably, CUR's antioxidative properties hold a great deal of potential for the treatment of depression. More and more evidences also showed that the activation of Nrf2 was the main mechanism of CUR in the treatment of oxidant stress-related diseases [26, 27]. Shen et al. have reported that CUR exerts its chemopreventive effects via the induction of antioxidant enzymes by activating Nrf2-ARE signaling [28]. Oxidative stress in the diabetic rat-induced by STZ could be attenuated by CUR through activation of the Keap1-Nrf2-ARE signaling pathway [29]. In addition, CUR augments the cardioprotective effect of metformin in an experimental model of type I diabetes mellitus via the Nrf2/HO-1 pathway which is also reported in previous study [30]. However, the detailed mechanism underlying the antidepressant effects of CUR as related to Nrf2 in the brain remains seldom studied.

Therefore, in our present study, we aimed to investigate the antidepressant-like effect of CUR in CUMS-induced rats. In addition, to further investigate the possible molecular mechanisms underlying the therapeutic effects of CUR, we also assessed whether the possible antidepressant-like effects of CUR are associated with oxidative stress status and the changes on the activation of Nrf2 in the brain.

\section{Materials and Methods}

2.1. Animals. Male Sprague-Dawley rats (180-220 g) were provided by the Hunan Cancer Hospital Animal Centre. The rats were housed in standard conditions $\left(23 \pm 2^{\circ} \mathrm{C}, 12 \mathrm{~h}\right.$ light/dark cycle). Except prior to sucrose preference test (SPT), food and water were freely available in the whole experiment. This study was approved by the Animal Care and Use Committee of Hunan Cancer Hospital (protocol number 016/2018). All experiments were performed in
TABLE 1: Specific modeling methods of CUMS

\begin{tabular}{lc}
\hline Number & CUMS procedure \\
\hline 1 & $24 \mathrm{~h}$ food deprivation \\
2 & $24 \mathrm{~h}$ water deprivation \\
3 & $45^{\circ}$ cage tilting for $24 \mathrm{~h}$ \\
4 & Restraint for $4 \mathrm{~h}$ in an empty water bottle \\
5 & 20 min of noise \\
6 & 1 min tail clamping \\
7 & Damp bedding \\
\hline
\end{tabular}

accordance with the Guide for Care and Use of Laboratory Animals (Chinese Council).

2.2. CUMS Procedure. The rats were adapted for one week and the CUMS procedure was performed for four weeks as previously described [31]. $24 \mathrm{~h}$ food deprivation followed by $24 \mathrm{~h}$ water deprivation, $45^{\circ}$ cage tilting for $24 \mathrm{~h}$, restraint for $4 \mathrm{~h}$ in an empty water bottle, $20 \mathrm{~min}$ of noise, $1 \mathrm{~min}$ tail clamping, and damp bedding were selected as stressors in our study. All stressors were applied individually, continuously, and randomly, so that the stress procedure is unpredictable. The detailed information for specific modeling methods of CUMS are shown in Table 1.

2.3. Experimental Design. Rats were randomly divided into three groups $(n=8)$ : control, CUMS, and CUMS+CUR. CUR (suspended in $0.5 \%$ Tween 80 , purchased from Sigma Chemical Co., USA) was administrated by oral gavage $(100 \mathrm{mg} / \mathrm{kg} /$ day) in the CUMS+CUR group, and the rats in control group were treated with the same volume of saline.

At the end of four weeks, behavioral tests were carried out, and the rats were sacrificed under anesthesia with an intraperitoneal injection of $1 \%$ sodium pentobarbital $(50 \mathrm{mg} / \mathrm{kg})$. Blood samples and the hippocampus were collected in our study. The whole experimental protocols are shown in Figure 1.

\subsection{Behavioral Test}

2.4.1. SPT. The SPT was performed as our previous study [32]. Prior to testing conditions, all the rats were separated in 1 cage each and habituated to $48 \mathrm{~h}$ of forced $1 \%$ sucrose solution consumption in two bottles on each side. After deprivation of water for $14 \mathrm{~h}$, two preweighted bottles containing $1 \%$ sucrose solution and tap water were given to each rat. After $1 \mathrm{~h}$, the bottles were weighed again, and the consumed weights of $1 \%$ sucrose solution and tap water were recorded. The percentage preference for sucrose was calculated as follows: sucrose preference $(\%)=$ sucrose consumption/ (sucrose consumption + water consumption).

2.4.2. Forced Swimming Test (FST). The FST was performed as described previously [33] with minor modifications. In brief, rats were separated and were forced to swim in an open cylindrical container ( $45 \mathrm{~cm}$ height, $25 \mathrm{~cm}$ diameter) containing $35 \mathrm{~cm}$ of water $\left(24 \pm 1^{\circ} \mathrm{C}\right)$ for a $15 \mathrm{~min}$ pretest. The rats were then dried and removed from their cages. 24 hours later, the rats were exposed to the same experimental conditions 


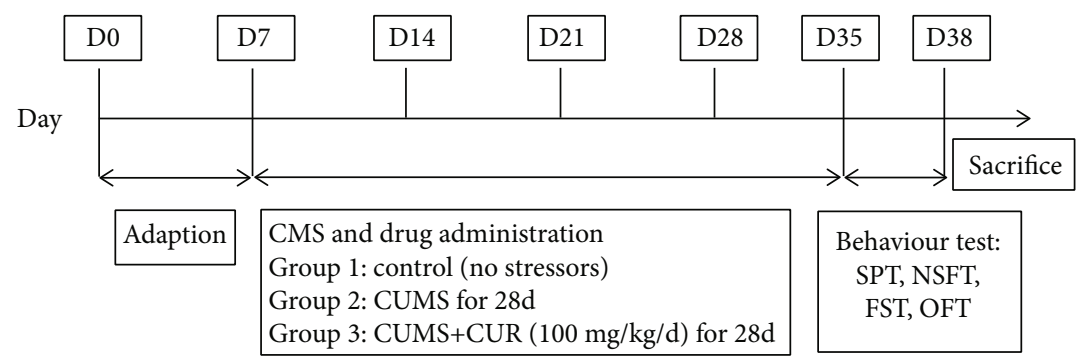

FIGURE 1: Schematic representation of experimental protocol.

TABle 2: Primers used in real-time PCR analyses of mRNA expression.

\begin{tabular}{lrc}
\hline Gene & Sense primer $\left(5^{\prime}-3^{\prime}\right)$ & Antisense primer $\left(5^{\prime}-3^{\prime}\right)$ \\
\hline HO-1 & TGCTCGCATGAACACTCTGGAGAT & ATGGCATAAATTCCCACTGCCACG \\
NQO- 1 & GTGAGAAGAGCCCTGATTGT & CCTGTGATGTCGTTTCTGGA \\
Nrf2 & CCCAGCACATCCAGACAG & TATCCAGGGCAAGCGACT \\
$\beta$-Actin & CATCCTGCGTCTGGACCTGG & TAATGTCACGCACGATTTCC \\
\hline
\end{tabular}

outlined above for a 5 min FST. Immobility time was scored by an experienced observer blind to the experiment design, defined as floating with only small movement necessary to keep the head above water.

2.4.3. Novelty-Suppressed Feeding Test (NSFT). All the rats were food deprived for $24 \mathrm{~h}$ in their home cages before NSFT. A piece of white paper $(10 \times 10 \mathrm{~cm})$ was placed in an open field $(75 \times 75 \times 40 \mathrm{~cm})$, and a small amount of food was placed on this paper. The rats were allowed to explore the open field for $8 \mathrm{~min}$. The time it took for the rat to approach and take the first bite of the food was defined as the latency time and was recorded in our study. Immediately afterwards, the animals were transferred to their home cage, and the total food intake for the next $5 \mathrm{~min}$ was also weighed to avoid the influence of the animals' appetite.

2.4.4. Open Field Test (OFT). The open field apparatus consisted of a $76 \times 76 \mathrm{~cm}$ gray wooden box with $42 \mathrm{~cm}$ high boundary walls. The floor was divided into 25 equal squares by black lines. Each rat was placed in the center of the square and left to explore it freely for $5 \mathrm{~min}$. The number of crossing and rearing was recorded by the observer blind to the treatment condition of the animal. The apparatus was cleaned with ethanol and water to remove olfactory cues.

2.5. Determination of Serum Corticosterone. For the determination of serum corticosterone, the blood samples were collected at 13:00-15:00 on day 38 before sacrifice. The collected plasma was centrifuged $(3500 \times \mathrm{g}, 15 \mathrm{~min})$ at $4^{\circ} \mathrm{C}$ and stored at $-80^{\circ} \mathrm{C}$ until analysis. The serum corticosterone levels were measured using a commercial ELISA kit (Cayman Chemical, USA) according to the manufacturer's instructions. The standards and samples were all run in duplicates, and the averaged data were used for statistical analysis.

2.6. Real-Time PCR Analysis. According to the instruction of manufactory, total RNA was extracted from the hippocampus using TRIzol reagent (Invitrogen Corp., Carlsbad, CA,
USA). The mRNA expression of Nrf2, NQO-1, and HO-1 was determined in our present study. Quantitative PCR was performed on Bio-Rad Cx96 Detection System (Bio-Rad, Hercules, CA, USA) using the SYBR green PCR kit (Applied Biosystems Inc., Woburn, MA, USA) and gene-specific primers. The sequences of gene-specific primers are listed in Table 2. A $5 \mathrm{ng}$ cDNA sample was used with 40 cycles of amplification. Each cDNA was determined in triplicate. The signals were normalized to $\beta$-actin as an internal standard.

2.7. Determination of Antioxidant Enzyme Activities and Lipid Peroxidation. Malondialdehyde (MDA) content was determined according to the previous report [34]. Briefly, $1 \mathrm{ml}$ of $15 \%$ trichloroacetic acid was added to $500 \mu \mathrm{l}$ of brain homogenate supernatant and mixed well, and then, the solutions were centrifuged at $1006 \times \mathrm{g}$ for $10 \mathrm{~min}$. One milliliter of the supernatant was added to $0.5 \mathrm{ml}$ of $0.7 \% \mathrm{TBA}$, and then, the mixture was heated for $60 \mathrm{~min}$ at $90^{\circ} \mathrm{C}$. The absorbance was recorded at $532 \mathrm{~nm}$ using UV spectrophotometer. CAT activity was assayed by $\mathrm{H}_{2} \mathrm{O}_{2}$ consumption, following Aebi's [35].

2.8. Western Blot Analysis. For western blot analysis, total protein was prepared from the hippocampus, and the Bradford method was used to determine its concentration. The hippocampus sample was loaded on a precast $12 \%$ SDS-PAGE gel with $10 \mu \mathrm{g}$ proteins in each lane. Proteins in the gels were transferred to a polyvinylidene fluoride membrane and blocked for $1 \mathrm{~h}$ in 5\% nonfat dry milk in TBS-T (25 mM Tris, pH 7.5, $150 \mathrm{mM} \mathrm{NaCl}, 0.05 \%$ Tween-20). The antibodies and concentrations listed below were used overnight at a temperature of $4^{\circ} \mathrm{C}$ : Nox2 (Santa Cruz; sc130549; 1:800); 4-HNE (Abcam ab48506; 1:1000); Nrf2 (Abcam; ab137550; 1:200); pCREB (Cell Signaling; 9198; $1: 1000$ ); CREB (Cell Signaling; 9197; 1:1000); BDNF (Abcam; ab108319; 1:2000); PSD-95 (ProteinTech; 206651-AP; 1:2000); synaptophysin (ProteinTech; 17785-1-AP; $1: 1000$ ); PCNA (ProteinTech; 10205-2-AP; $1: 3000$ ); and 


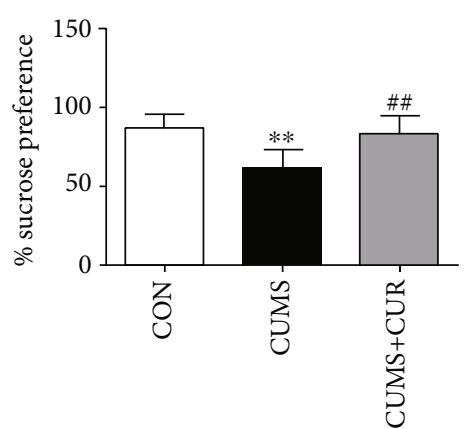

(a)

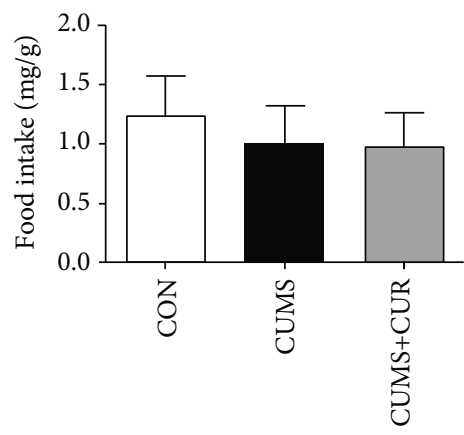

(d)

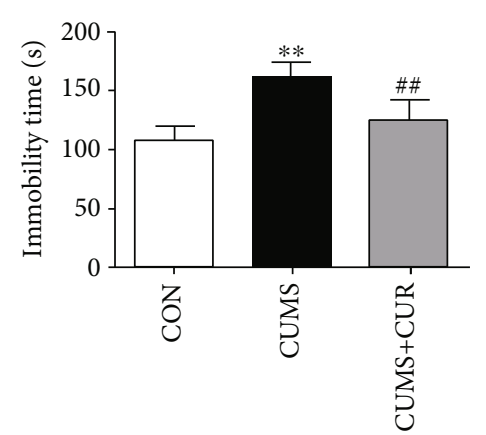

(b)

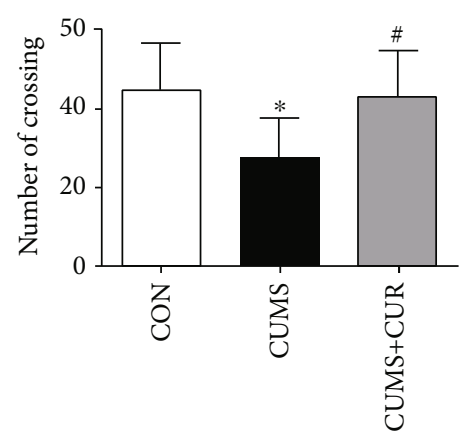

(e)

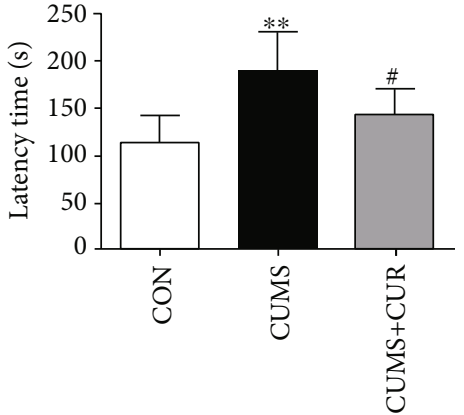

(c)

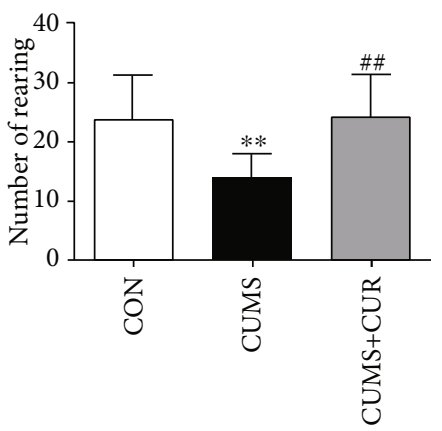

(f)

FIGURE 2: Effect of CUR on CUMS-induced behavior changes. (a) Sucrose preference in SPT, (b) immobility time in FST, (c) latency time in NSFT, (d) food intake in NSFT, (e) number of crossing in OPT, and (f) number of rearing in OPT. Data are expressed as means \pm SD $(n=8)$. $* p<0.05$ and $* * p<0.01$ compared to the control group. ${ }^{*} p<0.05$ and ${ }^{\# \#} p<0.01$ compared to the CUMS group.

$\beta$-actin (ProteinTech; 60008-1-Ig; $1: 4000)$. Membranes were then probed with horseradish peroxidase-conjugated secondary antibody for $40 \mathrm{~min}$. After washing, the membranes were dipped in electrochemiluminescence, and immunoblots were analyzed by using the Bioprofl Biolight PC software (Vulber Lourmat, France). $\beta$-Actin was used as an internal standard to normalize the signals.

2.9. TUNEL Staining. According to the manufacturer's instructions, the terminal deoxynucleotidyl transferasemediated deoxyuridine triphosphate nick-end labeling (TUNEL) detection kit (KeyGen Biotech, Nanjing, China) was used to assess apoptosis. Apoptotic index was defined as the average percentage of TUNEL-positive cells in 20 nonoverlapping cortical fields under $\times 200$ magnification.

2.10. Immunohistochemical Staining. Paraffin-embedded tissue sections were rehydrated first in xylene and then in graded ethanol solutions. The slides were then blocked with $5 \%$ bovine serum albumin (BSA) in Tris-buffered saline (TBS) for $2 \mathrm{~h}$. After incubation with anti-8-OHDG and anti-Nox 2 overnight at $4^{\circ} \mathrm{C}$, the sections were then washed with PBS and incubated with secondary antibodies. Counter staining was performed using hematoxylin, and the slides were visualized under a light microscope.

2.11. Golgi Staining. Golgi staining was performed as previous report [36]. In brief, the brain tissues of the rat were kept in the Golgi-Cox solution for 14 days in the darkness, and the solution was replaced every $48 \mathrm{~h}$. After dehydration with $30 \%$ sucrose solution, the tissues were cut into $100 \mu \mathrm{m}$ section. The following steps included treatment with ammonia water and acid hardening fixing bath and dehydration with increasing concentrations of alcohol. A digital camera attached with microscopy was used to take images of the tissues for dendritic structure analyzing. Dendritic spine density and total dendritic length analysis were done manually using the Fiji software under $\times 400$ magnification.

2.12. DHE Staining. Reactive oxygen species (ROS) was measured by dihydroethidium (DHE) microfluorography as previous study [37]. In brief, freshly prepared frozen brain sections $(15 \mu \mathrm{m}$ thick) were incubated with $5 \mu \mathrm{M}$ DHE in PBS at $37^{\circ} \mathrm{C}$ for $30 \mathrm{~min}$ in a dark humidified chamber. The sections were then imaged by using the Leica fluorescence microscope (Leica Microsystems, Germany).

2.13. Statistical Analysis. Statistical Package for Social Science (SPSS) version 18 (SPSS Inc., Chicago, IL, USA) was used for data analysis in our study. All data were analyzed by one-way analysis of variance (ANOVA) with least significant difference (LSD) post hoc multiple comparisons. All data were presented as means $\pm \mathrm{SD}$, and $p<0.05$ was considered statistically significant.

\section{Results}

3.1. Effects of CUR on Behavioral Tests. The CUMS group showed reduced source preference in SPT (Figure 2(a), $p<0.01$ ), prolonged immobility time in FST (Figure 2(b), 
$p<0.01$ ), and latency time in NSFT (Figure 2(c), $p<0.01$ ) in comparison with the rats in control group. Our study also observed that the number of crossing (Figure 2(e), $p<0.05$ ) and rearing (Figure $2(\mathrm{f}), p<0.01$ ) in OPT was all significantly decreased in the CUMS group. In comparison with the CUMS group, the administration of CUR successfully increased the sucrose preference (Figure 2(a), $p<0.01$ ), decreased immobility time (Figure $2(\mathrm{~b}), p<0.01$ ) and latency time (Figure 2(c), $p<0.01$ ), and increased the number of crossing (Figure $2(\mathrm{e}), p<0.05)$ and rearing (Figure 2(f), $p<0.01$ ) in the CUMS+CUR group. In addition, no significant difference of food intake was observed in NSFT.

3.2. Effects of CUR on Corticosterone Level. As displayed in Figure 3, the serum corticosterone level significantly increased $(p<0.01)$ in the CUMS group compared with the control group. However, the administration of CUR markedly decreased $(p<0.01)$ the corticosterone level when compared with the rats in the CUMS group.

3.3. Effect of CUMS and CUR on Oxidative Stress. The immunohistochemical staining results of 8-OHDG and Nox2 are shown in Figure 4(a); the results showed that the expressions of 8-OHDG and Nox2 were all increased in CUMS-treated rats when compared to control group, and the supplementation of CUR markedly moderated CUMS-induced increasing of 8-OHDG and Nox2. The results of DHE immunostaining showed that ROS production was significantly increased in the CUMS group, and this increase in ROS generation was markedly alleviated by the pretreatment with CUR (Figure 4(a)). The protein expressions of Nox2 (Figure 4(b), $p<0.01$ ) and 4 -HNE (Figure 4(c), $p<0.01$ ) were significantly increased in the CUMS group as compared to the rats in the control group, and the administration of CUR effectively mitigated CUMS-induced increasing of Nox2 (Figure 4(b), $p<$ 0.05 ) and 4-HNE (Figure 4(c, $p<0.01$ ); the western blot result of Nox2 was in accordance with immunohistochemical staining results. The content of MDA (Figure $4(\mathrm{~d}), p<0.01$ ) was significantly increased in the CUMS group when compared to the control group. The CUR treatment successfully decreased the content of MDA in the CUMS+CUR group when compared to the CUMS group (Figure 4(d), $p<0.01$ ). In comparison with the rats in the control group, the activity of CAT (Figure 4(e), $p>0.05$ ) was decreased in CUMStreated rats but without significance difference. Furthermore, the administration of CUR significantly increased CAT activity in the CUMS+CUR group when compared to the CUMS group (Figure $4(\mathrm{e}), p<0.05)$.

3.4. Effects of CUR on the Activation of Nrf2 in CUMSTreated Rats. The Nrf2 levels in cytoplasmic (Figure 5(a), $p<0.05$ ) and nuclear (Figure 5(b), $p<0.01$ ) all significantly decreased in the CUMS group when compared with the rats in the normal-treated group, and the Nrf2 in nuclear obviously (Figure $5(\mathrm{~b}), p<0.01$ ) increased in the CUMS+CUR group compared to the CUMS group. Interestingly, as shown in Figure 5(c), the gene expression of Nrf2 was similar with the protein expression of $\mathrm{Nrf2}$ in the

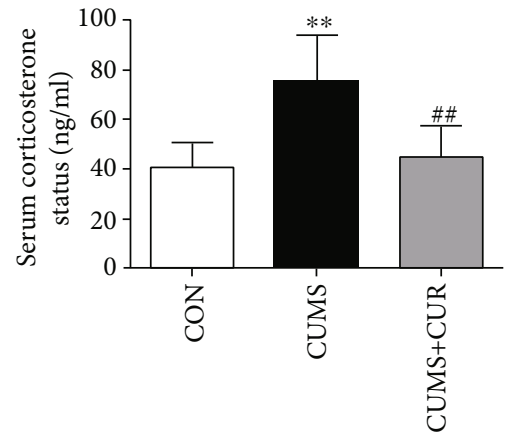

Figure 3: Effect of CUR on CUMS-induced serum corticosterone level. Data are expressed as means $\pm \mathrm{SD}(n=8) . * p<0.05$ and $* * p$ $<0.01$ compared to the control group. ${ }^{\#} p<0.05$ and ${ }^{\# \#} p<0.01$ compared to the CUMS group.

nuclear. The mRNA expressions of NQO-1 (Figure 5(d), $p<0.01$ ) and HO-1 (Figure 5(e), $p<0.05$ ) all significantly decreased in the CUMS model rats, and CUR treatment significantly prevented the decrease of NQO-1 (Figure 5(d), $p<0.01$ ) and HO-1 (Figure 5(e), $p<0.05$ ) in the CUMS+ CUR group when compared with the rats in the CUMS group.

3.5. Effect of CUMS and CUR on Synaptic Plasticity. As shown in Figure 6(b), the pCREB/CREB ratio was significantly decreased in the CUMS group compared to vehicle control group (Figure 6(b), $p<0.05$ ). Administration of CUR significantly increased pCREB/CREB ratio in the hippocampus compared to the CUS-treated rats (Figure 6(b), $p<0.01$ ). The protein expression of BDNF (Figure 6(c), $p<0.01$ ), PSD-95 (Figure 6(d), $p<0.05$ ), and synaptophysin (Figure 6(e), $p<0.01$ ) all significantly decreased in the CUMS-treated rats, and CUR successfully reversed the CUMS-induced decrease of these three proteins $(p<0.01)$. Previous studies have reported that dynamic alterations in synaptic and dendritic structure and function play a pivotal role in the development of depression $[38,39]$. In our present study, Golgi staining showed that spine density (Figure 6(g), $p<0.01$ ) and total dendritic length (Figure 6(i), $p<0.01$ ) significantly decreased in the dentate gyrus (DG) granule neurons of CUMS-induced rats, and the administration of CUR markedly reversed this effect (Figure $6(\mathrm{~g}), p<0.01$, Figure 6(i), $p<0.05$ ).

\section{Discussion}

Our present study demonstrated that the administration of CUR exhibited antidepressant-like activities in CUMSinduced depression model. We investigated the depressivelike behaviors (SFT, FST, NSFT, and OFT) in rats under CUMS, and chronic administration of CUR normalized behavioral changes in rats exposed to stress. CUR could effectively decrease protein expression of oxidative stress marker (MDA, Nox2, and 4-HNE). CUR could also activate stressinduced Nrf2-ARE axis inhibition. In addition, long-term treatment with CUR markedly prevented CUMS-induced reduction of BDNF, PSD-95, and synaptophysin expressions. 


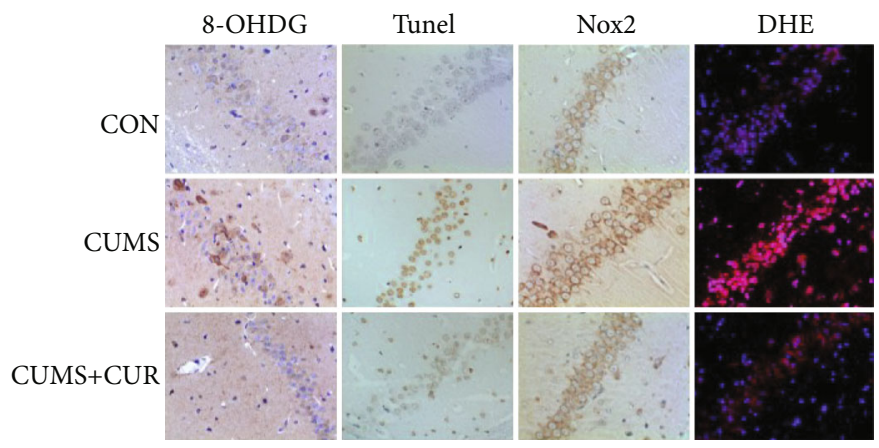

(a)

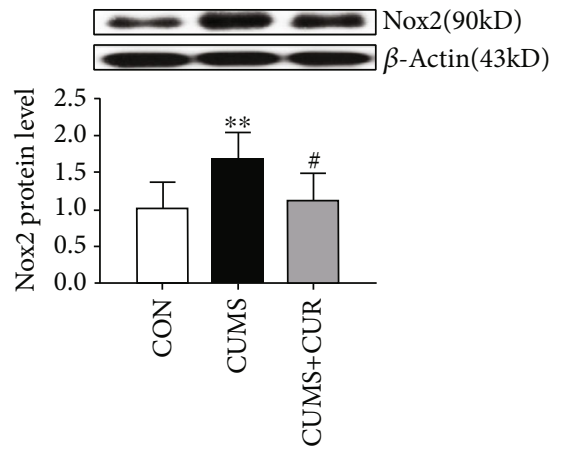

(b)

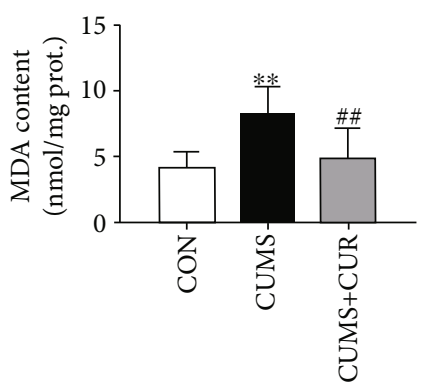

(d)

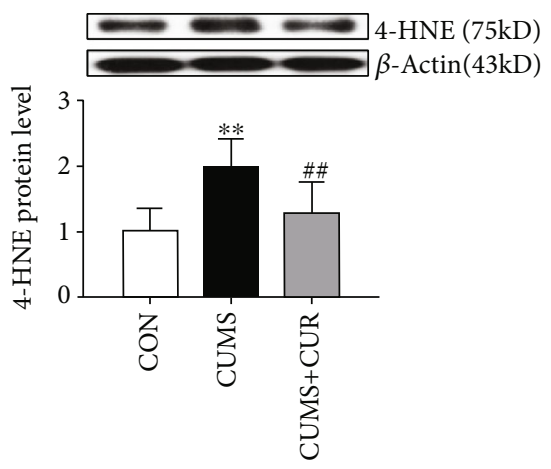

(c)

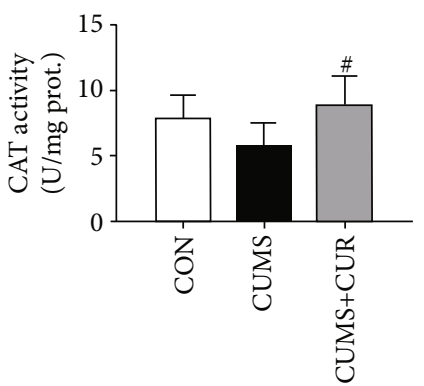

(e)

FIGURE 4: Effects of CUR on CUMS-induced oxidative stress change. (a) Immunohistochemical staining, TUNEL staining, and DHE staining, (b) protein expression of Nox2, (c) protein expression of 4-HNE, (d) content of MDA, and (e) activity of CAT. Data are expressed as means \pm SD $(n=8) . * p<0.05$ and $* * p<0.01$ compared to the control group. ${ }^{\#} p<0.05$ and ${ }^{\# \#} p<0.01$ compared to the CUMS group.

These findings indicate the potential benefits of administration of CUR to reverse the development of depression. Furthermore, the antidepressant mechanism of CUR may be mediated by restoring changes in oxidative stress and the activation of the Nrf2-ARE signaling pathway.

The CUMS model has long been used as animal model of depression, and previous study showed that most effects of CUMS could be effectively reversed by antidepressant agents [40]. In our present study, reduced sucrose preference in SPT and prolonged immobility time in FST were observed in the CUMS group, which indicated the depressive-like state. In comparison with the rats in the CUMS group, chronic administration of CUR successfully increased sucrose preference and decreased immobility time, which was consistent with the former studies $[41,42]$. Anxiety status was assessed by NSFT and OFT. Our study observed that CUR treatment could successfully reverse CUMS-induced increase in latency time in NSFT and decrease in crossing number and rearing number in OFT. Motaghinejad et al. have also reported that chronic administration of CUR could effectively improve ambulation number and ambulation distance in nicotinetreated rats, indicating neuroprotective effect of CUR against nicotine-induced neurotoxicity [43]. Therefore, CUR exhibited antidepressant-like properties basing on the abovementioned results.

As a stress marker, corticosterone level is widely used for accessing the stress state. More and more evidences showed that the elevated corticosterone level was associated with depressive-like behaviors, and antidepressant-like activity could always induce a reduction of corticosterone level [44-46]. In the present study, the corticosterone serum level was significantly increased in rats exposed to CUMS model compared to the nonstressed group, and chronic administration of CUR successfully reversed CUMSinduced the elevation of the corticosterone serum level. The results that serum corticosterone in the rats of the CUMS 


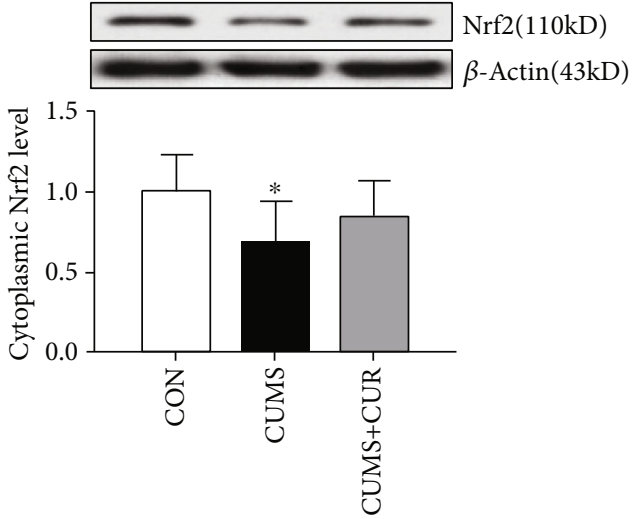

(a)

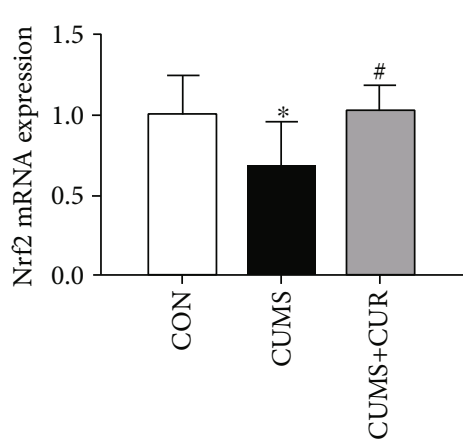

(c)

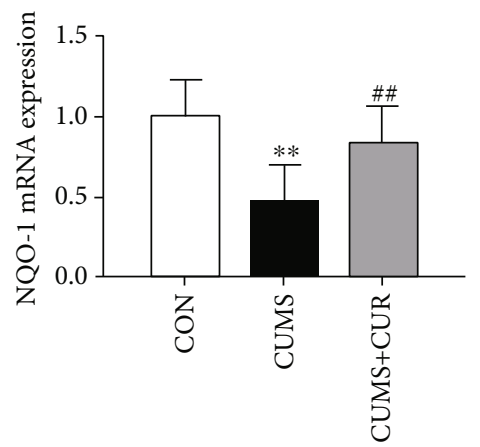

(d)

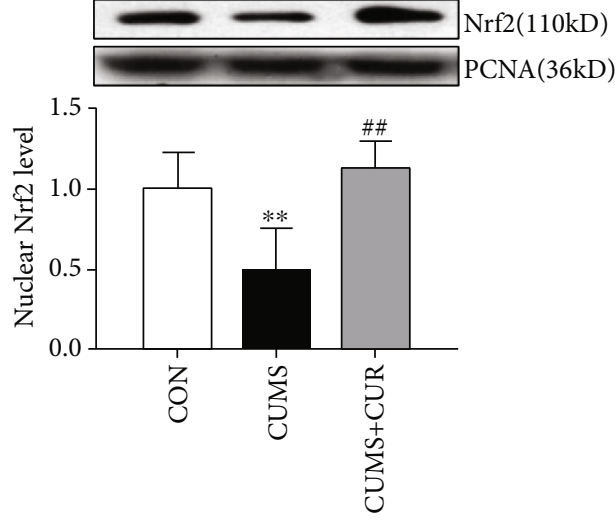

(b)

FIGURE 5: Effects of CUR on the activation of Nrf2 in CUMS-treated rats. (a) Protein expression of Nrf2 in cytoplasmic, (b) protein expression of Nrf2 in nuclear, (c) mRNA expression of Nrf2 in the hippocampus, (d) mRNA expression of NOQ-1in the hippocampus, and (e) mRNA expression of HO- 1 in the hippocampus. Data are expressed as means $\pm \mathrm{SD}(n=8) . * p<0.05$ and $* * p<0.01$ compared to the control group. ${ }^{\#} p<0.05$ and ${ }^{\# \#} p<0.01$ compared to the CUMS group.

group increased significantly were in accordance with previous studies [2, 47]. As a typical antidepressant agent, fluoxetine could effectively prevent the elevation of serum corticosterone level in CUMS-treated rats [1, 48]. Disrupting the HPA axis and elevating the levels of serum corticosterone by CUMS were increasingly recognized $[49,50]$. In addition, CUMS-induced increase of serum corticosterone level may be due to an impaired negative feedback in the HPA $[51,52]$ and lack of the inhibitory role of the hippocampus in glucocorticoid synthesis [53].

The elevated free radical generation and decreased activity of antioxidants break the balance between oxidantantioxidant systems, which will always induce oxidative stress [54]. More and more evidence showed that the brain is highly susceptible to oxidative damage [55], and oxidative stress plays a pivotal role in CUMS-induced depression [54]. The release of excitatory amino acid and the expression of specific gene may be enhanced by ROS, which will always induce lipid peroxidation and DNA oxidation, subsequently resulting in neuronal apoptosis [56]. Our present study observed that the intensity of DHE staining and TUNELpositive cells significantly increased in CUMS-treated rats, which indicated oxidative stress induced a severe neuronal apoptosis. Fortunately, as a natural antioxidant agent, the administration of CUR significantly alleviated CUMSinduced oxidative stress and neuronal apoptosis in the
CUMS+CUR group. In our present study, signs of oxidative stress were observed as exemplified by the decrease of antioxidant enzyme activity, such as CAT. In addition, as lipid peroxidation markers, MDA and 4-HNE levels all significantly increased in the CUMS group when compared to the control group. Previous study has reported that CUR has shown to counteract oxidative stress by reducing lipid peroxidation and improving the activity of antioxidant enzymes [54]. Our study results were consistent with this report, which expressed a significantly decrease in MDA and 4-HNE levels and a markedly increase in CAT activity in the CUMS+CUR group. Furthermore, DNA is an important and recognized target of free radicals attack. 8-OHDG is one of the most widely studied biomarkers of oxidative DNA damage. The immunohistochemical staining results coincide with a previous study, which indicated that CUR effectively reversed the increase of 8-OHDG expression under CUMS. ROS and oxidative stress are mainly generated from Nox which is a multiunit enzyme [57]. In particular, the primary mechanism underlying the development of oxidative stress in various neurodegenerative conditions is the activation of Nox2 [58]. In our present study, the protein expression of Nox2 significantly increased in CUMS-treated rats, and the administration of CUR successfully alleviated this phenomenon in the CUMS+CUR group. These results supported that oxidative stress plays a pivotal role in CUMS-induced depression. 


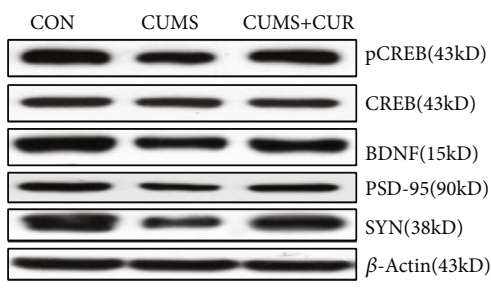

(a)

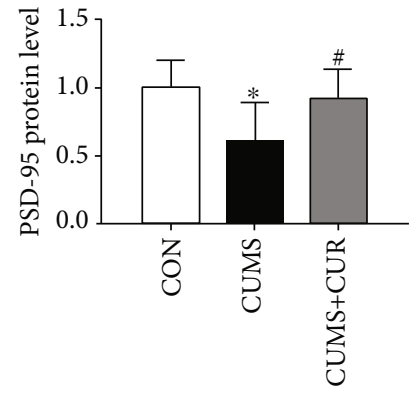

(d)

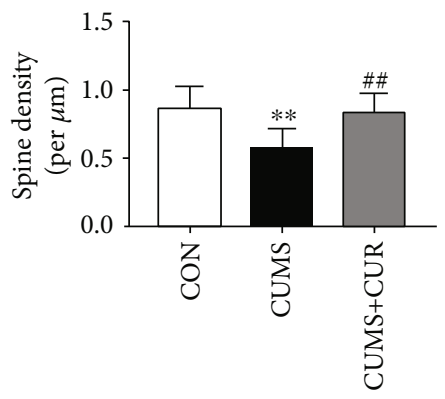

(g)

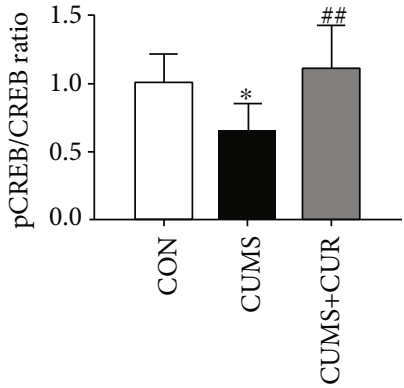

(b)

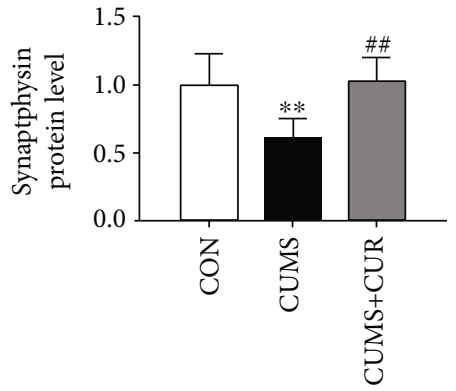

(e)

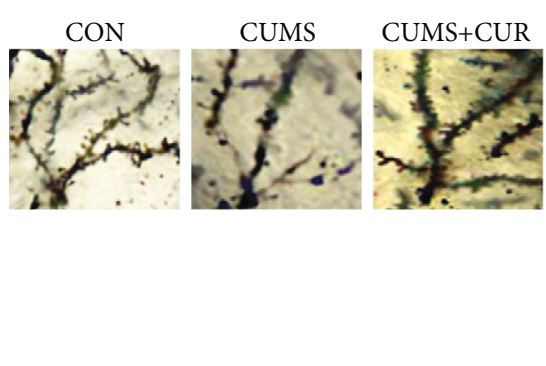

(h)

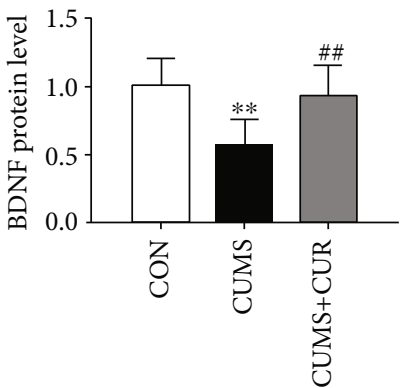

(c)

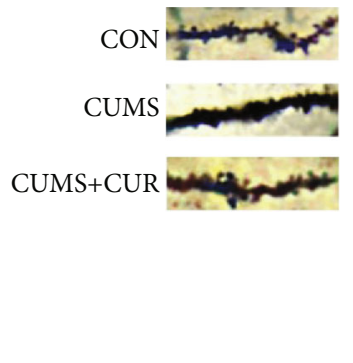

(f)

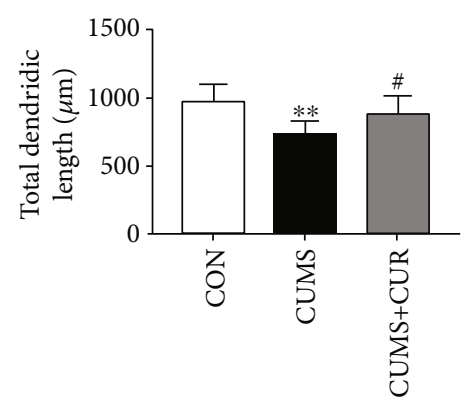

(i)

FIGURE 6: Effect of CUR on synaptic plasticity. (b) pCREB/CREB ratio in the hippocampus, (c) protein expression of BDNF in the hippocampus, (d) protein expression of PSD-95 in the hippocampus, (e) protein expression of synaptophysin in the hippocampus, (f) representative images of dendritic spines from DG granule neurons in the rat, (g) spine density in different groups, (h) representative images of DG granule neurons in the rats, and (i) total dendritic length in different groups. Data are expressed as means \pm SD $(n=8)$. $* p$ $<0.05$ and $* * p<0.01$ compared to the control group. ${ }^{\#} p<0.05$ and ${ }^{\# \#} p<0.01$ compared to the CUMS group.

In addition, our results also demonstrated that the neuroprotective effect of CUR was mediated via its antioxidative ability.

As a transcription factor, Nrf2 is known to play a pivotal role in modulating oxidative stress and exhibiting an important protective role in brain injury and neurodegenerative diseases. As it is known to us, Nrf2 is mainly located in the cytoplasm under physical conditions. However, Nrf2 translocates into the nucleus in response to oxidative stress [15]. In our present study, the results of western blot showed that CUR administration promoted Nrf2 nuclear translocation. This indicates that the Nrf2 level markedly increased in CUMS+CUR-treated rats when compared with the rats in the CUMS group. And the protein expression of Nrf2 was without significant difference in both the CUMS+CUR group and the CUMS group. The above observations revealed that Nrf2 was inhibited when rats exposed to CUMS and CUR had the ability to activate Nrf2. Previous studies have also reported that CUR had the ability to activate $\mathrm{Nrf} 2$ and provide neuroprotection from a traumatic brain injury $[16,56]$. In order to investigate the regulation effects of CUR on the Nrf2 downstream pathway, the mRNA levels of Nrf2, NQO-1, and HO-1 were evaluated in our study. NQO-1 and HO-1 are important antioxidant enzymes in the Nrf2-ARE pathway [59]. The results showed that chronic administration of CUR could significantly reverse CUMSinduced decrease in the mRNA level of Nrf2, NQO-1, and HO- 1 . The above results indicated that the Nrf2 downstream pathway was inhibited under CUMS, and chronic administration of CUR could effectively activate this signal pathway. Our results indicated that the Nrf2 signal pathway was inhibited under CUMS, and chronic administration of CUR enhanced Nrf2 translocation from cytoplasm to nucleus and increased expression of antioxidant enzymes through 
Nrf2 signal pathway, thereby protecting the brain against CUMS-induced depression.

CREB is involved in the regulation of genes associated with synaptic and neural plasticity [43]. CREB has been reported to be phosphorylated by many signaling events on serine 133 [60]. The results in our study showed that the ratio of pCREB/CREB significantly decreased in the CUMS group, and chronic administration of CUR successfully reversed this reduction. These results were in conformity with previous studies which have shown that CUR can increase the pCREB/CREB ratio in rats exposed to CUMS [61]. In addition, a previous study also showed that CUR could function as a potential agent that suppresses depressive-like behavior via the prevention of protein changes associated with synaptic plasticity [62]. As the most abundant neurotrophin in the brain, BDNF plays a crucial role in the regulation of survival as well as synaptic plasticity. Our study investigated whether BDNF was involved in the antidepressant effects induced by CUR. The results indicated that the expression of BDNF in rats exposed to CUMS was significantly increased after chronic administration of CUR. Furthermore, other synapse-associated proteins were also accessed in our study. PSD-95 and synaptophysin were postsynaptic marker and presynaptic marker respectively. In accordance with BDNF, CUR successfully alleviated CMUS-induced reduction in the protein level of PSD-95 and synaptophysin. Zhang et al. have reported that CUR can reverse the decreased expression of BDNF, PSD-95, and synaptophysin in CMS-induced rats [62]. Previous studies have shown that spine densities and synaptic plasticity were closely correlated with the function of neuron and cognitive performance $[63,64]$. Our present study shows that CUR could effectively reverse CUMS-induced decrease of spine density and total dendritic length. Hence, the alteration of the above-mentioned synaptic plasticityassociated proteins may underlie changes in functional plasticity associated with CUMS-induced depression.

\section{Conclusion}

In conclusion, our present study suggests that the administration CUR is effective in preventing CUMS-induced depression. Furthermore, the current results suggested that the antidepressant action of CUR may be mediated by restoring changes in oxidative stress, the Nrf2-ARE signaling pathway, and the synaptic and neural plasticity, which might ultimately contribute to its antidepressive-like effect.

\section{Data Availability}

The data used to support the findings of this study are available from the corresponding author upon request.

\section{Conflicts of Interest}

The authors declare no conflict of interest.

\section{Authors' Contributions}

Pei Jiang and Ni Liu designed the study and wrote the protocol. Dehua Liao, Chuanfeng Lv, Lizhi Cao, Yi Wu, and Minghui Long performed the experiments and analyzed the data. Dunwu Yao contributed to the reagents and materials. Dehua Liao and Chuanfeng Lv drafted the manuscript. Pei Jiang and Dehua Liao revised the manuscript content. All authors read and approved the final manuscript. Dehua Liao and Chuanfeng Lv contributed equally to this manuscript.

\section{Acknowledgments}

This study was supported by the National Natural Science Foundation of China (NSFC: 81603206), Health and Family Planning Commission Foundation of Hunan Province (grant numbers B20180402 and C2019064), the Project of Hunan Provincial Science \& Technology Department (grant numbers 2019JJ80093 and 2020JJ8093), the Key Research and Development Program of Jining Science and Technology (2019SMNS012), the Traditional Chinese Medicine Science and Technology Development Plan of Shandong Province (2019-0747), and the Shandong Science and Technology Development Plan on Medicine and Hygiene in China (No. 2017WS348).

\section{References}

[1] Y. H. Kim, A. R. Im, B.-K. Park et al., "Antidepressant-like and neuroprotective effects of ethanol extract from the root bark ofHibiscus syriacusL," BioMed Research International, vol. 2018, Article ID 7383869, 13 pages, 2018.

[2] G.-F. Wu, S. Ren, R.-Y. Tang et al., “Antidepressant effect of taurine in chronic unpredictable mild stress-induced depressive rats," Scientific Reports, vol. 7, 2017.

[3] J. B. Potash, A. J. Ferrari, F. J. Charlson et al., "The epidemiological modelling of major depressive disorder: application for the Global Burden Of Disease Study 2010," PLoS One, vol. 8, article e69637, 2013.

[4] E. Holtzheimer Paul and NCB, "Advances in the treatment of depression,” NeuroRx, vol. 3, p. 14, 2006.

[5] D. Badyal, R. Deswal, A. Sharma, and P. Matreja, "Efficacy and safety of add on low-dose mirtazapine in depression," Indian Journal of Pharmacology, vol. 44, no. 2, pp. 173-177, 2012.

[6] R. Mojtabai, M. Olfson, N. A. Sampson et al., "Barriers to mental health treatment: results from the National Comorbidity Survey Replication," Psychological Medicine, vol. 41, no. 8, pp. 1751-1761, 2011.

[7] W. M.-L. Licinio Julio, "Depression, antidepressants and suicidality a critical appraisal," Nature Reviews. Drug Discovery, vol. 4, p. 6, 2005.

[8] C. Taylor, A. D. Fricker, L. A. Devi, and I. Gomes, "Mechanisms of action of antidepressants: from neurotransmitter systems to signaling pathways," Cellular Signalling, vol. 17, no. 5, pp. 549-557, 2005.

[9] V. N. Thakare, V. D. Dhakane, and B. M. Patel, "Potential antidepressant-like activity of silymarin in the acute restraint stress in mice: modulation of corticosterone and oxidative stress response in cerebral cortex and hippocampus," Pharmacological Reports, vol. 68, no. 5, pp. 1020-1027, 2016. 
[10] M. Gazal, M. R. Valente, B. A. Acosta et al., "Neuroprotective and antioxidant effects of curcumin in a ketamine-induced model of mania in rats," European Journal of Pharmacology, vol. 724, pp. 132-139, 2014.

[11] F. Ng, M. Berk, O. Dean, and A. I. Bush, "Oxidative stress in psychiatric disorders: evidence base and therapeutic implications," The International Journal of Neuropsychopharmacology, vol. 11, no. 6, 2008.

[12] M. Maes, P. Galecki, Y. S. Chang, and M. Berk, “A review on the oxidative and nitrosative stress (O\&NS) pathways in major depression and their possible contribution to the (neuro)degenerative processes in that illness," Progress in NeuroPsychopharmacology and Biological Psychiatry, vol. 35, no. 3, pp. 676-692, 2011.

[13] R. Chhillar and D. Dhingra, "Antidepressant-like activity of gallic acid in mice subjected to unpredictable chronic mild stress," Fundamental \& Clinical Pharmacology, vol. 27, no. 4, pp. 409-418, 2013.

[14] L. Cigliano, M. S. Spagnuolo, F. Boscaino et al., "Dietary supplementation with fish oil or conjugated linoleic acid relieves depression markers in mice by modulation of the Nrf2 pathway," Molecular Nutrition \& Food Research, vol. 63, article e1900243, 2019.

[15] A. Kobayashi, M. I. Kang, H. Okawa et al., "Oxidative stress sensor Keap1 functions as an adaptor for Cul3-based E3 ligase to regulate proteasomal degradation of Nrf2," Molecular and Cellular Biology, vol. 24, no. 16, pp. 7130-7139, 2004.

[16] W. Dong, B. Yang, L. Wang et al., "Curcumin plays neuroprotective roles against traumatic brain injury partly via Nrf2 signaling," Toxicology and Applied Pharmacology, vol. 346, pp. 28-36, 2018.

[17] H. E. de Vries, M. Witte, D. Hondius et al., "Nrf2-induced antioxidant protection: a promising target to counteract ROS-mediated damage in neurodegenerative disease?," Free Radical Biology and Medicine, vol. 45, no. 10, pp. 1375-1383, 2008.

[18] W. Li and A.-N. Kong, "Molecular mechanisms of Nrf2mediated antioxidant response," Molecular Carcinogenesis, vol. 48, no. 2, pp. 91-104, 2009.

[19] I. Buendia, P. Michalska, E. Navarro, I. Gameiro, J. Egea, and R. Leon, "Nrf2-ARE pathway: an emerging target against oxidative stress and neuroinflammation in neurodegenerative diseases," Pharmacology \& Therapeutics, vol. 157, pp. 84104, 2016.

[20] S. H. Christiansen, M. V. Olesen, G. Wortwein, and D. P. Woldbye, "Fluoxetine reverts chronic restraint stress-induced depression-like behaviour and increases neuropeptide $\mathrm{Y}$ and galanin expression in mice," Behavioural Brain Research, vol. 216, no. 2, pp. 585-591, 2011.

[21] M. Popoli, Z. Yan, B. S. McEwen, and G. Sanacora, "The stressed synapse: the impact of stress and glucocorticoids on glutamate transmission," Nature Reviews. Neuroscience, vol. 13, no. 1, pp. 22-37, 2011.

[22] A. C. Conti, J. F. Cryan, A. Dalvi, I. Lucki, and J. A. Blendy, "cAMP response element-binding protein is essential for the upregulation of brain-derived neurotrophic factor transcription, but not the behavioral or endocrine responses to antidepressant drugs," The Journal of Neuroscience, vol. 22, no. 8, pp. 3262-3268, 2002.

[23] B. B. Aggarwal and K. B. Harikumar, "Potential therapeutic effects of curcumin, the anti-inflammatory agent, against neurodegenerative, cardiovascular, pulmonary, metabolic, auto- immune and neoplastic diseases," The International Journal of Biochemistry \& Cell Biology, vol. 41, no. 1, pp. 40-59, 2009.

[24] R. K. Maheshwari, A. K. Singh, J. Gaddipati, and R. C. Srimal, "Multiple biological activities of curcumin: a short review," Life Sciences, vol. 78, no. 18, pp. 2081-2087, 2006.

[25] Y. Xu, B.-S. Ku, H.-Y. Yao et al., "The effects of curcumin on depressive-like behaviors in mice," European Journal of Pharmacology, vol. 518, no. 1, pp. 40-46, 2005.

[26] C. Yang, X. Zhang, H. Fan, and Y. Liu, "Curcumin upregulates transcription factor $\mathrm{Nrf} 2, \mathrm{HO}-1$ expression and protects rat brains against focal ischemia," Brain Research, vol. 1282, pp. 133-141, 2009.

[27] I. S. Carmona-Ramírez, A. Tobón-Velasco, J. C. Orozco-Ibarra et al., "Curcumin restores Nrf2 levels and prevents quinolinic acid-induced neurotoxicity," The Journal of Nutritional Biochemistry, vol. 24, pp. 14-24, 2013.

[28] X. C. Shen Guoxiang, H. Rong, R. Jain Mohit et al., "Modulation of nuclear factor E2-related factor 2-mediated gene expression in mice liver and small intestine by cancer chemopreventive agent curcumin," Molecular Cancer Therapeutics, vol. 5, pp. 39-51, 2006.

[29] Z. Xie, B. Wu, G. Shen, X. Li, and Q. Wu, "Curcumin alleviates liver oxidative stress in type 1 diabetic rats," Molecular Medicine Reports, vol. 17, pp. 103-108, 2017.

[30] E. M. Abdelsamia, S. A. Khaleel, A. Balah, and N. A. Abdel Baky, "Curcumin augments the cardioprotective effect of metformin in an experimental model of type I diabetes mellitus; impact of Nrf2/HO-1 and JAK/STAT pathways," Biomedicine \& Pharmacotherapy, vol. 109, pp. 2136-2144, 2019.

[31] G. G. Ducottet Cecile and B. Catherine, "Effects of the selective nonpeptide corticotropin-releasing factor receptor 1 antagonist antalarmin in the chronic mild stress model of depression in mice," Progress in Neuro-Psychopharmacology \& Biological Psychiatry, vol. 27, p. 6, 2003.

[32] D. Liao, D. Xiang, R. Dang et al., "Neuroprotective effects of dl3-n-butylphthalide against doxorubicin-induced neuroinflammation, oxidative stress, endoplasmic reticulum stress, and behavioral changes," Oxidative Medicine and Cellular Longevity, vol. 2018, Article ID 9125601, 13 pages, 2018.

[33] S. Kumar and A. C. Mondal, "Neuroprotective, neurotrophic and anti-oxidative role of Bacopa monnieri on CUS induced model of depression in rat," Neurochemical Research, vol. 41, no. 11, pp. 3083-3094, 2016.

[34] H. O. N. Ohkawa and K. Yagi, "Assay for lipid peroxides in animal tissues by thiobarbituric acid reaction," Analytical Biochemistry, vol. 95, p. 7, 1979.

[35] H. Aebi, "Catalase in vitro," Methods in Enzymology, vol. 105, p. $5,1984$.

[36] N. Abe-Higuchi, S. Uchida, H. Yamagata et al., "Hippocampal sirtuin 1 signaling mediates depression-like behavior," Biological Psychiatry, vol. 80, no. 11, pp. 815-826, 2016.

[37] J. Mo, B. Enkhjargal, Z. D. Travis et al., “AVE 0991 attenuates oxidative stress and neuronal apoptosis via Mas/PKA/CREB/UCP-2 pathway after subarachnoid hemorrhage in rats," Redox Biology, vol. 20, pp. 75-86, 2019.

[38] R. S. Duman and G. K. Aghajanian, "Synaptic dysfunction in depression: potential therapeutic targets," Science, vol. 338, no. 6103, pp. 68-72, 2012.

[39] V. Krishnan and E. J. Nestler, "The molecular neurobiology of depression," Nature, vol. 455, no. 7215, pp. 894-902, 2008. 
[40] P. Willner, "Validity, reliability and utility of the chronic mild stress model of depression a 10-year review and evaluation," Psychopharmacology, vol. 134, no. 4, pp. 319-329, 1997.

[41] Y.-C. Li, F.-M. Wang, Y. Pan et al., "Antidepressant-like effects of curcumin on serotonergic receptor-coupled AC-cAMP pathway in chronic unpredictable mild stress of rats," Progress in Neuro-Psychopharmacology and Biological Psychiatry, vol. 33, no. 3, pp. 435-449, 2009.

[42] M. K. Bhutani, M. Bishnoi, and S. K. Kulkarni, "Anti-depressant like effect of curcumin and its combination with piperine in unpredictable chronic stress-induced behavioral, biochemical and neurochemical changes," Pharmacology Biochemistry and Behavior, vol. 92, no. 1, pp. 39-43, 2009.

[43] M. Motaghinejad, M. Motevalian, S. Fatima, F. Faraji, and S. Mozaffari, "The neuroprotective effect of curcumin against nicotine-induced neurotoxicity is mediated by CREB-BDNF signaling pathway," Neurochemical Research, vol. 42, no. 10, pp. 2921-2932, 2017.

[44] M. E. Crupi Rosalia, M. Angela, L. S. Giuseppina, B. Placido, C. Salvatore, and S. Edoardo, "Melatonin treatment mimics the antidepressant action in chronic corticosterone-treated mice," Journal of Pineal Research, vol. 49, p. 6, 2010.

[45] B. Lee, I. Shim, H. J. Lee, Y. Yang, and D. H. Hahm, "Effects of acupuncture on chronic corticosterone-induced depressionlike behavior and expression of neuropeptide $\mathrm{Y}$ in the rats," Neuroscience Letters, vol. 453, no. 3, pp. 151-156, 2009.

[46] Y. Zhao, W. Xie, J. Dai, Z. Wang, and Y. Huang, “The varying effects of short-term and long-term corticosterone injections on depression-like behavior in mice," Brain Research, vol. 1261, pp. 82-90, 2009.

[47] N. Jiang, B.-Y. Zhang, L.-M. Dong et al., "Antidepressant effects of dammarane sapogenins in chronic unpredictable mild stress-induced depressive mice," Phytotherapy Research, vol. 32, no. 6, pp. 1023-1029, 2018.

[48] V. N. Thakare, R. R. Patil, R. J. Oswal, V. D. Dhakane, M. K. Aswar, and B. M. Patel, "Therapeutic potential of silymarin in chronic unpredictable mild stress induced depressive-like behavior in mice," Journal of Psychopharmacology, vol. 32, pp. 223-235, 2017.

[49] Z. Ma, G. Wang, L. Cui, and Q. Wang, "Myricetin attenuates depressant-like behavior in mice subjected to repeated restraint stress," International Journal of Molecular Sciences, vol. 16, no. 12, pp. 28377-28385, 2015.

[50] W. Quan, F. Liu, Y. Zhang et al., "Antidepressant-like effects of magnesium lithospermate $B$ in a rat model of chronic unpredictable stress," Pharmaceutical Biology, vol. 53, no. 8, pp. 1168-1175, 2015.

[51] J. G. Tasker and J. P. Herman, "Mechanisms of rapid glucocorticoid feedback inhibition of the hypothalamic-pituitaryadrenal axis," Stress, vol. 14, no. 4, pp. 398-406, 2011.

[52] M. Keller-Wood, "Hypothalamic-pituitary-adrenal axis-feedback control," Comprehensive Physiology, vol. 5, pp. 1161-1182, 2015.

[53] H. F. Sakr, A. M. Abbas, A. Z. Elsamanoudy, and F. M. Ghoneim, "Effect of fluoxetine and resveratrol on testicular functions and oxidative stress in a rat model of chronic mild stress-induced depression," Journal of Physiology and Pharmacology, vol. 66, p. 12, 2015.

[54] S. Samarghandian, M. Azimi-Nezhad, T. Farkhondeh, and F. Samini, "Anti-oxidative effects of curcumin on immobilization-induced oxidative stress in rat brain, liver and kidney," Biomedicine \& Pharmacotherapy, vol. 87, pp. 223-229, 2017.

[55] M. Motaghinejad, M. Motevalian, and B. Shabab, "Effects of chronic treatment with methylphenidate on oxidative stress and inflammation in hippocampus of adult rats," Neuroscience Letters, vol. 619, pp. 106-113, 2016.

[56] W. Dai, H. Wang, J. Fang et al., "Curcumin provides neuroprotection in model of traumatic brain injury via the Nrf2ARE signaling pathway," Brain Research Bulletin, vol. 140, pp. 65-71, 2018.

[57] L. M. Fan, S. Cahill-Smith, L. Geng, J. Du, G. Brooks, and J.-M. Li, "Aging-associated metabolic disorder induces Nox2 activation and oxidative damage of endothelial function," Free Radical Biology and Medicine, vol. 108, pp. 940-951, 2017.

[58] S. J. Cooney, S. L. Bermudez-Sabogal, and K. R. Byrnes, "Cellular and temporal expression of NADPH oxidase (NOX) isotypes after brain injury," Journal of Neuroinflammation, vol. 10, p. 13, 2013.

[59] Y. Xie, Q. Y. Zhao, H. Y. Li, X. Zhou, Y. Liu, and H. Zhang, "Curcumin ameliorates cognitive deficits heavy ion irradiation-induced learning and memory deficits through enhancing of Nrf2 antioxidant signaling pathways," Pharmacology Biochemistry and Behavior, vol. 126, pp. 181-186, 2014.

[60] R. Wang, Y.-H. Li, Y. Xu et al., "Curcumin produces neuroprotective effects via activating brain-derived neurotrophic factor/TrkB-dependent MAPK and PI-3K cascades in rodent cortical neurons," Progress in Neuro-Psychopharmacology and Biological Psychiatry, vol. 34, no. 1, pp. 147-153, 2010.

[61] Y. Xu, B. Ku, L. Tie et al., "Curcumin reverses the effects of chronic stress on behavior, the HPA axis, BDNF expression and phosphorylation of CREB," Brain Research, vol. 1122, no. 1, pp. 56-64, 2006.

[62] L. Zhang, J. Luo, M. Zhang, W. Yao, X. Ma, and S. Y. Yu, "Effects of curcumin on chronic, unpredictable, mild, stressinduced depressive-like behaviour and structural plasticity in the lateral amygdala of rats," The International Journal of Neuropsychopharmacology, vol. 17, no. 5, pp. 793-806, 2014.

[63] J. I. Luebke, C. M. Weaver, A. B. Rocher et al., "Dendritic vulnerability in neurodegenerative disease: insights from analyses of cortical pyramidal neurons in transgenic mouse models," Brain Structure \& Function, vol. 214, no. 2-3, pp. 181-199, 2010.

[64] S. T. Tsai, S. Y. Chen, S. Z. Lin, and G. F. Tseng, "Rostral intralaminar thalamic deep brain stimulation ameliorates memory deficits and dendritic regression in beta-amyloid-infused rats," Brain Structure \& Function, vol. 225, no. 2, pp. 751-761, 2020. 